\title{
Basal Autonomic Activity, Stress Reactivity, and Increases in Metabolic Syndrome Components over Time.
}

Mandy X. Hu ${ }^{\mathrm{a}}$, MD, Femke Lamers ${ }^{\mathrm{a}}$, PhD, Sarah A. Hiles ${ }^{\mathrm{a}}$, PhD, Brenda W. J. H. Penninx ${ }^{\mathrm{a}}$, PhD, Eco J.C. de Geus ${ }^{\mathrm{b}}$, PhD

aDepartment of Psychiatry and EMGO Institute for Health and Care Research, VU University Medical Centre, AJ Ernststraat 1187, 1081 HL Amsterdam, The Netherlands

${ }^{\text {b}}$ Department of Biological Psychology and EMGO Institute for Health and Care Research, VU University, van der Boechorststraat 1, 1081 BT Amsterdam, The Netherlands

Abbreviated Title: Autonomic functioning and the metabolic syndrome

Word count: 3891

Number of figures and tables: 5

Corresponding author and person to whom reprint requests should be addressed:

Mandy Xian Hu

Department of Psychiatry

VU University Medical Centre

AJ Ernststraat 1187, 1081 HL Amsterdam, the Netherlands

Telephone: (+31) 020-7884521

E-mail address: $\underline{\text { m.hu@ggzingeest.nl }}$ 


\begin{abstract}
Context: Basal autonomic nervous system (ANS) functioning has been linked to the metabolic syndrome (MetS), but the role of ANS reactivity in response to stress remains unclear.
\end{abstract}

Objective: To examine cross-sectionally and longitudinally to what extent ANS basal level and stress reactivity are related to MetS.

Design: 2-year and 6-year data from a prospective cohort: the Netherlands Study of Depression and Anxiety.

Setting: Participants were recruited from the general community, primary care, and mental health care organizations.

Participants: 1922 respondents (mean age=43.7 years).

Main outcome measures: Indicators of ANS functioning were heart rate (HR), respiratory sinus arrhythmia (RSA) and pre-ejection period (PEP). ANS stress reactivity was measured during a cognitively challenging stressor and a personal-emotional stressor. MetS components included triglycerides, high-density lipoprotein cholesterol, blood pressure, glucose and waist circumference.

Results: Cross-sectional analyses indicated that higher basal HR, lower basal values of RSA and PEP, and higher RSA reactivity during cognitive challenge were related to less favorable values of almost all individual MetS components. Longitudinal analyses showed that higher basal HR and shorter basal PEP predicted 4-year increase in many MetS abnormalities. Higher RSA stress reactivity during cognitive challenge predicted 4-year increase in number of MetS components.

Conclusion: Higher basal sympathetic, lower basal parasympathetic activity, and increased parasympathetic withdrawal during stress are associated with multiple MetS components, and higher basal sympathetic activity predicts an increase in metabolic abnormalities over time. These findings support a role for ANS dysregulation in the risk for MetS and, consequently, the development of cardiovascular disease. 
Mandy Xian Hu

Keywords: Autonomic nervous system, metabolic syndrome, stress, cardiovascular disease 


\section{Introduction}

Cardiovascular disease (CVD) is the most important cause of disability-adjusted life years according to the World Health Organization (2009). The autonomic nervous system (ANS) plays a central role in cardiovascular regulation (Ulrich-Lai and Herman, 2009) but is also implicated in the etiology of CVD through its effects on the metabolic syndrome (MetS), a cluster of risk factors for CVD (Eckel et al., 2005). According to current biological understanding, innervation of the ANS modulates glucose and fat metabolism (Kyrou and Tsigos, 2009; Romijn and Fliers, 2005), liver metabolism (Yi et al., 2010), and virtually all other components of daily energy expenditure (Lambert et al., 2010; Thorp and Schlaich, 2015). Indeed, many studies have linked basal ANS dysregulation to high waist circumference, blood pressure (BP), triglycerides, glucose, and/or low high-density lipoprotein (HDL) cholesterol (Brunner et al., 2002; Grassi et al., 2005; Kimura et al., 2006; Koskinen et al., 2009; Liao et al., 1998; Licht et al., 2010; Ljung et al., 2000; Min et al., 2008; Stein et al., 2007; Vrijkotte et al., 2015). In a cross-sectional study with 1883 participants, Licht et al. (2010) found that increased sympathetic (SNS) and decreased parasympathetic nervous system (PNS) activity were associated with MetS. Furthermore, Vrijkotte and colleagues (2015) showed that the association between this ANS imbalance and an unfavorable metabolic profile is already present in children at the age of 5 to 6 years. Longitudinal studies in this research field suggested that basal plasma norepinephrine predicted 5-year increases in weight and blood pressure (Masuo et al., 2003), and that dysregulated basal ANS level predicted 2-year increase in metabolic abnormalities (Licht et al., 2013).

In addition to chronic elevations of basal ANS level, there is reason to believe that ANS reactivity to psychological stressors is implicated in MetS. In response to a threat, a shift toward SNS dominance and PNS withdrawal is seen, and it is suggested that exaggeration of this response leads to metabolic alterations (Hjemdahl, 2002; Kyrou and Tsigos, 2009; Lambert and Lambert, 2011). Some studies have linked elevated cardiovascular responses to stress with increased central adiposity, increased BP, and elevated levels of cholesterol and/or triglycerides (Fisher and Newman, 2013; Goldbacher et al., 2005; Jorgensen et al., 1988; Muldoon, 1995; Nyklíček et al., 2005; Steptoe et al., 1996; Tuomisto, 1997). However, these studies used increases in HR and BP as indicators of stress 
reactivity. The association between autonomic stress reactivity of both the SNS and the PNS with the entire cluster of MetS components remains underexposed in the literature, as do longitudinal studies to infer directionality. Some longitudinal studies have linked higher cardiovascular reactivity in response to stress to the development of hypertension (Carroll et al., 2001; Light et al., 1992; Matthews and Salomon, 2003; Matthews et al., 2004; Menkes et al., 1989; Ming et al., 2004; Steptoe and Cropley, 2000). Again, in these studies stress reactivity was mainly assessed by increased BP. Flaa et al. (2008) showed that sympathetic activity during mental arithmetic predicted 18 year increase in blood pressure. However, this was only the case for absolute values during the stress task and not for stress reactivity values. To our knowledge, only one previous study aimed to determine the predictive value of autonomic responses to psychological laboratory stress for development of metabolic burden (Gentile et al., 2015). The results indicated that parasympathetic withdrawal in response to stress was associated with an increased metabolic burden in men, but not women.

Given the scarcity of empirical data on this important theoretical link, this large-scale study aimed to 1) examine cross-sectionally to what extent ANS basal level and stress reactivity are independently related to MetS, and 2) establish the predictive value of ANS basal level and stress reactivity in the 4-year increase of MetS components. Indicators of ANS activity were: 1) heart rate (HR), an index of both parasympathetic and sympathetic cardiac control (De Geus and Van Doornen, 1996), 2) respiratory sinus arrhythmia (RSA), an index of cardiac vagal control (De Geus and Van Doornen, 1996; de Geus et al., 1995; Neijts et al., 2014), and 3) pre-ejection period (PEP), an index of left ventricular sympathetic control (De Geus and Van Doornen, 1996; Lien et al., 2015). Stress reactivity was evoked by two types of stressors: 1) a cognitively challenging stressor (an n-back task) and 2) a personal-emotional stressor in the form of a psychiatric interview (earlier described by Hu et al. (Hu et al., in press)). We hypothesized that an unfavorable basal ANS profile, defined by high HR in the presence of low vagal control (low RSA) and high sympathetic control (short PEP), and ANS hyperreactivity to stress, characterized by a greater increase in HR and greater decrease in RSA and PEP, would be associated with unfavorable metabolic profiles and would predict an increase in metabolic abnormalities over time. 


\section{Methods}

\subsection{Subjects}

Data was obtained from the Netherlands Study of Depression and Anxiety (NESDA), an ongoing longitudinal cohort including 2981 respondents aged 18-65 years. Respondents were recruited from the community, primary care and mental health care in The Netherlands. A four-hour baseline measurement was conducted between September 2004 and February 2007, and follow-up assessments took place after two, four and six years. Demographic, psychiatric and physical assessments were included, as well as collection of blood. The two-year follow-up also included a cognitively challenging stressor (n-back task). A detailed description of the rationale, objectives and methods of the NESDA study can be found elsewhere (Penninx et al., 2008). The research protocol was approved by the Ethical Review Board of each participating center, and all respondents provided written informed consent.

For the cross-sectional part of the current study, data were drawn from the two-year follow-up assessment (in later sections referred to as baseline data), since the cognitively challenging stressor was not introduced until then. Of the total sample of 2596 respondents, 674 individuals were excluded because of missing physiological data due to equipment failure during assessment, poor electrocardiogram quality or because they did not complete one of the stress conditions. The crosssectional study sample therefore consisted of 1922 participants.

The longitudinal part of this study was conducted with the two-year follow-up data (baseline) and the six-year follow-up data (in later sections referred to as 4-year data). The longitudinal sample consisted of 2256 respondents of which 581 people were excluded because of missing physiological data, and another 59 individuals because of missing data on all MetS components. Consequently, the longitudinal analyses were conducted with 1616 participants.

\subsection{Physiological measurement}

During the assessments, the participants wore the 'Vrije Universiteit Ambulatory Monitoring System' (VU-AMS). The VU-AMS is an unobtrusive lightweight portable device that records 
electrocardiograms (ECG) and changes in thorax impedance (ICG) from a six-electrode configuration (De Geus and Van Doornen, 1996). During the recording the different assessment conditions were divided by an event marker. Movement registration through vertical accelerometry was used to remove periods where the subjects were not stationary.

The interbeat interval (IBI) time series were extracted from the ECG signal to obtain HR (Neijts et al., 2014). RSA combined the ECG with the respiration signal obtained from the thorax impedance, and was derived by subtracting the shortest IBI during HR acceleration at inhalation from the longest IBI during HR deceleration at exhalation for all breaths (De Geus and Van Doornen, 1996; de Geus et al., 1995; Neijts et al., 2014). PEP was defined as the interval between the Q-onset in the ECG, indicating onset of left ventricular electrical activity, and the upstroke (B-point) of the ICG signal, indicating the beginning of left ventricular ejection (De Geus and Van Doornen, 1996; Lien et al., 2015).

Suspicious IBIs and breathing cycles were corrected or discarded during automated and visual data cleaning. An automated scoring algorithm was also used to detect crucial landmarks in the ICG, which were then visually inspected and manually corrected.

\subsection{Stress conditions}

As described in more detail before (Hu et al., in press), the participants were exposed to two different stress conditions. The order of exposure was first a psychiatric interview, evoking personal-emotional stress, and second the n-back task, representing the 'classical' cognitively challenging tasks often used in laboratory settings.

\subsubsection{Psychiatric interview stressor}

The psychiatric interview of approximately 45 minutes included questions regarding various indicators of anxious and depressive symptoms, as well as suicidal thoughts, mood disorder symptoms and experience of adverse life events. The participants were asked to describe and recall these negative experiences extensively.

\subsection{2. $N$-back task stressor}


A spatial variant of the n-back task was administered for which the participants were required to remember the location of a stimulus presented a certain number ' $n$ ' of stimuli back. Three n-back conditions (1-back, 2-back and 3-back) were presented to the participants, each consisting of 20 trials. There were two components which made this task stress-evoking: task difficulty and performance pressure on the one hand, and on the other hand the provision of false feedback and negative comparison to other participants.

\subsubsection{Basal level}

For the psychiatric interview stressor, a general interview served as a basal level. The general interview lasted approximately 30 minutes and included topics such as somatic health, smoking behavior, use of medication, daily functioning and health care utilization.

Prior to the n-back task, an n-back rest was administered during which participants were presented with a series of neutral pictures for 2 minutes. The n-back rest was used as a basal level to compare against the n-back task stressor.

\subsection{Metabolic syndrome components}

MetS was defined according to the American Heart Association and National Heart, Lung and Blood Institute's update of the U.S. National Cholesterol Education Program-Adult Treatment Panel III criteria (Grundy et al., 2005). The number of components was determined by the presence of the following criteria: 1) waist circumference $\geq 102 \mathrm{~cm}$ in men and $\geq 88 \mathrm{~cm}$ in women, 2) $\mathrm{SBP} \geq 130$ and/or DBP $\geq 85 \mathrm{~mm} \mathrm{Hg}$ or antihypertensive medication; 3) triglycerides $\geq 1.7 \mathrm{mmol} / \mathrm{L}$ or medication for hypertriglyceridemia; 4) HDL cholesterol $<1.03 \mathrm{mmol} / \mathrm{L}$ in men and $<1.30 \mathrm{mmol} / \mathrm{L}$ in women or medication for reduced HDL cholesterol; 5) fasting plasma glucose $\geq 5.6 \mathrm{mmol} / \mathrm{L}$ or antidiabetic medication.

Waist circumference was measured with a measuring tape at the central point between the lowest front rib and the highest front point of the pelvis. SBP was measured twice on the right arm during supine rest with Omron M4-I, HEM 752A, and averaged over the two measurements. 
Triglycerides, HDL cholesterol and glucose were determined by collecting blood samples in the morning after an overnight fast.

The continuous MetS components were adjusted for medication use based on estimated average effects of the medication (Grundy et al., 2005). For persons using antihypertensive medication, 10 mm Hg was added to their SBP values (Cui et al., 2003; Licht and Geus, 2009; Mancia and Parati, 2004; SHEP Cooperative Research Group, 1991; Tannen et al., 2006; Vogelzangs and Suthers, 2007). For persons using fibrates, $0.67 \mathrm{mmol} / \mathrm{L}$ was added to triglycerides and $0.10 \mathrm{mmol} / \mathrm{L}$ was subtracted from HDL cholesterol. In addition, when using nicotinic acid $0.19 \mathrm{mmol} / \mathrm{L}$ was added to triglycerides and $0.15 \mathrm{mmol} / \mathrm{L}$ was subtracted from HDL cholesterol (Bays et al., 2003; Grundy et al., 2005). Subjects using antidiabetic medication were assigned a value of $7.0 \mathrm{mmol} / \mathrm{L}$ when glucose level was less than $7.0 \mathrm{mmol} / \mathrm{L}$ (Vogelzangs and Suthers, 2007).

\subsection{Covariates}

Adjustments were made for sociodemographic characteristics, including age, sex and years of education. We additionally included smoking status (yes/no) as a health confounder. Since it has been linked to RSA (Hirsch and Bishop, 1981; Mortara et al., 1997), respiration rate was also included as a covariate.

\subsection{Statistical analyses}

ANS basal values were obtained by taking the average score of n-back rest and the general interview. Absolute stress reactivity values for n-back were calculated by subtraction of ANS values during the n-back rest from ANS values during the n-back task. Similarly, absolute stress reactivity values for interview were calculated by subtraction of ANS values during the general interview from ANS values during the psychiatric interview.

Adjusted multiple linear regression analyses were used to analyze the cross-sectional association of basal ANS levels and stress reactivity values with the number of MetS components and continuous individual MetS components. Since triglycerides and glucose levels were non-normally distributed, these components were ln-transformed before analyses. Multiple linear regression was 
also used to investigate the longitudinal relationship between ANS (re)activity and changes in number of MetS components and changes in continuous individual MetS components over time. For these longitudinal analyses, baseline metabolic values were taken into account in addition to the other covariates. Both cross-sectional and longitudinal analyses were conducted in two steps: a first model including only basal HR, RSA or PEP levels, and a second model adding stress reactivity values. Sensitivity analyses were performed with additional adjustment for current and remitted depressive and anxiety disorder, and for antidepressant use. Regular use of the following antidepressants were included: tricyclic antidepressants (TCAs, ATC code N06AA), selective serotonin reuptake inhibitors (SSRIs, ATC code N06AB), and selective serotonin and noradrenalin reuptake inhibitors (SNRIs, ATC code N06AX). We also investigated possible sex modification by adding a sex-by-ANS value interaction to the analyses. The criterion for statistical significance was $\mathrm{p}<.05$. Data was analyzed using IBM SPSS Statistics for Windows, version 20.0 (IBM Corp). 


\section{Results}

\subsection{Cross-sectional associations between ANS and MetS components}

Table 1 shows that our sample ( $n=1922)$ had a mean age of 43.7 years $( \pm 13.2 S D)$ and $66.5 \%$ were female.

Table 2 shows that several ANS measures were significantly associated with MetS components. Of the basal ANS values (model 1), higher HR was associated with higher levels of triglycerides $(\beta=.152)$, SBP $(\beta=.118)$, glucose $(\beta=.108)$, larger waist circumference $(\beta=.106)$, and higher number of MetS components $(\beta=.128)$, all $p$-values $<.001$. Lower basal RSA (indicative of decreased PNS activity) and shorter basal PEP (indicative of increased SNS activity) were associated with higher levels of triglycerides ( $\beta=-.059, \mathrm{p}=.027$ and $\beta=-.090, \mathrm{p}<.001$, respectively), SBP $(\beta=-.079$, $\mathrm{p}=.001$ and $\beta=-.120, \mathrm{p}<.001$, respectively), larger waist circumference $(\beta=-.054, \mathrm{p}=.024$ and $\beta=-.141$, $\mathrm{p}<.001$, respectively), and higher number of MetS components $(\beta=-.071, \mathrm{p}=.005$ and $\beta=-.127, \mathrm{p}<.001$, respectively). In addition, shorter basal PEP was significantly related to lower HDL cholesterol $(\beta=.055, \mathrm{p}=.009)$.

We graphically illustrated the association between basal RSA and PEP and the number of MetS components, categorized as 0 components, 1 or 2 components, and 3 or more components. Figure 1 shows that a higher number of MetS components was associated with lower (normalized and adjusted) values of both basal RSA and PEP.

The addition of ANS stress reactivity measures to the analyses (model 2) showed that mainly reactivity to the cognitively challenging stressor was associated with MetS components. Unexpectedly, higher HR reactivity to cognitive challenge was related to higher HDL cholesterol ( $\beta=.056, \mathrm{p}=.012)$ and lower waist circumference $(\beta=-.055, \mathrm{p}=.008)$. However, RSA and PEP reactivity showed results in the expected direction. Higher RSA reactivity to cognitive challenge was associated with higher levels of triglycerides $(\beta=-.091, \mathrm{p}<.001)$, SBP $(\beta=-.071, \mathrm{p}=.002)$, higher waist circumference $(\beta=-.089, \mathrm{p}<.001)$, and higher number of MetS components $(\beta=-.102, \mathrm{p}<.001)$. Higher PEP reactivity to a cognitively challenging stressor was related to higher SBP $(\beta=-.040, \mathrm{p}=.039)$. Some associations with metabolic abnormalities were also found for ANS reactivity to the personal- 
emotional stressor. For this stressor, higher RSA reactivity was related to higher glucose levels ( $\beta=-$ $.051, \mathrm{p}=.023)$ and higher waist circumference $(\beta=-.051, \mathrm{p}=.017)$, whereas higher PEP reactivity was related to lower levels of HDL cholesterol $(\beta=.044, \mathrm{p}=.046)$.

\subsection{Longitudinal associations between ANS and changes in MetS components}

Table 3 shows that over the 4-year follow-up period, a mean increase was seen in triglycerides, HDL cholesterol, SBP, glucose, waist circumference and number of metabolic components.

Longitudinal associations between ANS measures and changes in MetS components (Table 4) were less prominent than the cross-sectional relationships. However, basal ANS values (model 1) significantly predicted 4-year increases in metabolic abnormalities. Higher HR values predicted a decrease in HDL cholesterol $(\beta=-.052, \mathrm{p}=.038)$ and an increase in $\operatorname{SBP}(\beta=.059, \mathrm{p}=.016)$ and number of MetS components $(\beta=.062, p=.016)$. Lower basal RSA only predicted increased SBP $(\beta=-.070$, $\mathrm{p}=.015)$. Shorter basal PEP predicted increased triglycerides $(\beta=-.050, \mathrm{p}=.028), \operatorname{SBP}(\beta=-.051$, $p=.034)$, waist circumference $(\beta=-.061, p=.015)$, and number of MetS components $(\beta=-.068, p=.007)$.

When adding ANS stress reactivity values to the analyses (model 2), only one significant association was found: higher RSA reactivity during cognitive challenge was associated with an increased number of MetS components $(\beta=-.069, \mathrm{p}=.020)$.

\subsection{Additional analyses}

We performed sensitivity analyses to additionally adjust for current and remitted depressive and anxiety disorder, as well as for the use of TCA, SSRI and SNRI. The results remained comparable after the adjustments, suggesting that neither psychopathology status or antidepressant use were driving our findings (Supplementary table 1 and 2).

When testing for sex modification, the analyses generally yielded a stronger relationship between ANS basal level and stress reactivity with MetS components for women than for men. 
Mandy Xian Hu

\section{Discussion}

This large study demonstrated that an unfavorable ANS profile, characterized by higher basal values of HR and lower values of RSA and PEP, was cross-sectionally associated with less favorable values of almost all individual MetS components. Longitudinal follow-up showed that higher basal values of HR and lower basal values of PEP predicted 4-year increase in many MetS abnormalities. Stressinduced changes in ANS parameters, mainly decrease in RSA during cognitive challenge, were crosssectionally associated with less favorable MetS profiles, and longitudinally predicted an increase in number of MetS components.

Our cross-sectional results are largely in line with literature linking basal autonomic dysregulation to MetS (Brunner et al., 2002; Grassi et al., 2015, 2005; Koskinen et al., 2009; Liao et al., 1998; Min et al., 2008; Stein et al., 2007; Vrijkotte et al., 2015) and MetS components (Kimura et al., 2006; Ljung et al., 2000). Furthermore, our cross-sectional findings in the 2-year follow-up data collection of the NESDA study are consistent with those in the baseline wave (Licht et al., 2010) where associations between basal SNS and PNS values and MetS components of similar magnitude were found. In addition, our 4-year follow-up findings were congruent with those of the previously conducted 2-year follow-up study (Licht et al., 2013) that also suggested an important role of the SNS in the development of metabolic abnormalities. Lower vagal control significantly predicted an increase in BP but only a trend was seen toward lower PNS activity predicting worsening of other metabolic values over time. The reason why the PNS had a robust association with MetS in the crosssectional but not the longitudinal analysis remains unclear but could reflect lower power to detect effects on the change-scores in MetS components compared to effects on levels. Overall, our longer follow-up duration elicited stronger associations than the 2-year follow-up study of Licht and colleagues (Licht et al., 2013). This suggests that with longer follow-up duration more robust effects of the PNS on the development of MetS components are seen.

In addition to a strong replication of the effects of ANS basal levels we tested the added value of ANS reactivity in response to stress in explaining variance in the MetS components. Our findings were partly comparable to the results of Gentile and colleagues (2015) who found that 
parasympathetic withdrawal in response to psychological stress predicted increased metabolic burden. Interestingly, the results of Gentile et al. were mostly evident in men. When looking solely at women, a blunted responsiveness of the PNS predicted increased metabolic burden. In contrast, our results implied that the relationship between a hyperactive ANS and MetS components was mostly evident in women. These conflicting findings illustrate the current state of research on the link between stress reactivity and metabolic abnormalities yielding mixed results. For instance, one study found that HR acceleration during laboratory tasks was associated with higher serum lipids (Jorgensen et al., 1988), another study indicated that blunted HR reactivity in response to a cognitive task was associated with more central adiposity (Carroll et al., 2008), whereas our analyses did not yield convincing results for HR reactivity. Overall, our findings implicate a small added value of testing for exaggerated parasympathetic stress reactivity in addition to the basal levels when trying to explain individual differences in MetS. The value of stress reactivity might increase with larger absolute reactivity; the stressors used here evoked significant but rather modest ANS reactivity. More research is needed on SNS and PNS reactivity in response to different stressors to further our understanding of the relationship between ANS basal level, autonomic stress reactivity and MetS.

When interpreting our findings, some limitations have to be considered. First, the majority of the NESDA sample consists of people with (a history of) depression and/or anxiety disorder, and both disorders have been linked to ANS dysregulation (Carney et al., 2005; Fisher and Newman, 2013; Salomon et al., 2013; Scalco et al., 2009; Schmitz et al., 2011). Furthermore, a considerable number of our respondents were using antidepressants, and studies have shown that these might influence ANS activity (Barton et al., 2007; Licht et al., 2012, 2008). Therefore, our results may not be entirely generalizable to the general population. However, additional adjustments for both psychopathology status and antidepressant use did not change our findings, suggesting a genuine association between ANS activity and the MetS. Second, our personal-emotional stressor is not validated as a stress task in literature and this stressor is susceptible to interpersonal variability. However, previous research showed that this condition generally evoked an increased autonomic stress reactivity compared to basal ANS level (Hu et al., in press). Another limitation is that, compared to daily life stress, our short-term stressors likely induced a mild stress reactivity. However, stress reactivity during the 
psychiatric interview was equal to the reactivity during the n-back task, which in turn is similar to the magnitude of reactivity found in literature (Mehler et al., 2010). We note that our aim was to examine potential biological pathways contributing to the MetS, as opposed to providing a clinically useful marker of dysfunctional stress reactivity at the level of individual participants. Finally, we did not have data available yet to address the question of reverse causality, or to investigate how changes in ANS functioning are associated with changes in MetS components over time. These questions should be clarified by future research.

Our study also had several strengths. We focused on both cross-sectional and longitudinal associations of ANS dysregulation with metabolic alterations, confirming previous findings in literature and contributing evidence to a causal pathway. Multiple measures of ANS were used, indicating both SNS and PNS activity, and continuous MetS components adjusted for medication use were analyzed individually in addition to a combined MetS count variable. Also, this is one of few studies to investigate the effect of ANS stress reactivity on MetS. And, finally, our large sample size allowed us to adjust for several key confounding factors.

In summary, this study suggests that higher basal sympathetic and lower basal parasympathetic activity are associated with MetS. Stress-induced decreases in PNS further contribute to MetS. In the longitudinal follow-up study, higher basal SNS activity proved to be the best predictor of the 4-year increase in metabolic abnormalities. These findings support a role for ANS dysregulation in the risk for MetS and they are congruent with our biological understanding of the autonomic stress response mobilizing the body for action, amongst others by recruiting available energy resources. When stress becomes chronic, this prolonged defense reaction can lead to a shift in metabolism and have detrimental effects on cardiovascular health. Interventions restoring ANS balance, such as stress-reducing programs, might ameliorate metabolic abnormalities and thereby cardiovascular morbidity and mortality. 


\section{References}

Barton, D.A., Dawood, T., Lambert, E.A., Esler, M.D., Haikerwal, D., Brenchley, C., Socratous, F., Kaye, D., Schlaich, M., Hickie, I., Lambert, G., 2007. Sympathetic activity in major depressive disorder: identifying those at increased cardiac risk? J. Hypertens. 25, 2117-2124.

Bays, H., Dujovne, C., McGovern, M., 2003. Comparison of once-daily, niacin extendedrelease/lovastatin with standard doses of atorvastatin and simvastatin (the ADvicor Versus Other Cholesterol-Modulating. Am. J. ... 91, 667-672.

Brunner, E.J., Hemingway, H., Walker, B.R., Page, M., Clarke, P., Juneja, M., Shipley, M.J., Kumari, M., Andrew, R., Seckl, J.R., 2002. Adrenocortical, autonomic, and inflammatory causes of the metabolic syndrome nested case-control study. Circulation 106, 2659-2665.

Carney, R.M., Freedland, K.E., Veith, R.C., 2005. Depression, the autonomic nervous system, and coronary heart disease. Psychosom. Med. 67, S29-S33.

Carroll, D., Phillips, A.C., \& Der, G. (2008). Body mass index, obesity, abdominal adiposity and cardiovascular reactions to psychological stress in a large community sample., 2008. . Psychosom. Med. 70, 653-660.

Carroll, D., Smith, G., Shipley, M., 2001. Blood pressure reactions to acute psychological stress and future blood pressure status: a 10-year follow-up of men in the Whitehall II study. Psychosom. Med. 63, 737-743.

Cui, J., Hopper, J., Harrap, S., 2003. Antihypertensive treatments obscure familial contributions to blood pressure variation. Hypertension 41, 207-210.

De Geus, E.J.C., Van Doornen, L.J.P., 1996. Ambulatory assessment of parasympathetic/sympathetic balance by impedance cardiography, in: Ambulatory Assessment: Computer-Assisted Psychological and Psychophysiological Methods in Monitoring and Field Studies. pp. 141-163.

de Geus, E.J.C., Willemsen, G.H.M., Klaver, C.H.A.M., van Doornen, L.J.P., 1995. Ambulatory measurement of respiratory sinus arrhythmia and respiration rate. Biol. Psychol. 41, 205-227.

Eckel, R.H., Grundy, S.M., Zimmet, P.Z., 2005. The metabolic syndrome. Lancet 365, 1415-1428.

Fisher, A.J., Newman, M.G., 2013. Heart rate and autonomic response to stress after experimental 
induction of worry versus relaxation in healthy, high-worry, and generalized anxiety disorder individuals. Biol. Psychol. 93, 65-74.

Flaa, A., Eide, I.K., Kjeldsen, S.E., Rostrup, M., 2008. Sympathoadrenal stress reactivity is a predictor of future blood pressure: an 18-year follow-up study. Hypertension 52, 336-41. doi:10.1161/HYPERTENSIONAHA.108.111625

Gentile, C., Dragomir, A.I., Solomon, C., Nigam, A., D’Antono, B., 2015. Sex differences in the prediction of metabolic burden from physiological responses to stress. Ann. Behav. Med. 49, 112-27. doi:10.1007/s12160-014-9639-2

Goldbacher, E., Matthews, K., Salomon, K., 2005. Central adiposity is associated with cardiovascular reactivity to stress in adolescents. Heal. Psychol. 24, 375-384.

Grassi, G., Dell’Oro, R., Quarti-Trevano, F., Scopelliti, F., Seravalle, G., Paleari, F., Gamba, P.L., Mancia, G., 2005. Neuroadrenergic and reflex abnormalities in patients with metabolic syndrome. Diabetologia 48, 1359-65. doi:10.1007/s00125-005-1798-z

Grassi, G., Mark, A., Esler, M., 2015. The sympathetic nervous system alterations in human hypertension. Circ. Res. 116, 976-990.

Grundy, S., Vega, G., Yuan, Z., 2005. Effectiveness and tolerability of simvastatin plus fenofibrate for combined hyperlipidemia (the SAFARI trial). Am. J. Cardiol. 95, 462-468.

Grundy, S.M., Cleeman, J.I., Daniels, S.R., Donato, K.A., Eckel, R.H., Franklin, B.A., Gordon, D.J., Krauss, R.M., Savage, P.J., Smith, S.C., Spertus, J.A., Costa, F., 2005. Diagnosis and management of the metabolic syndrome: an American Heart Association/National Heart, Lung, and Blood Institute Scientific Statement. Circulation 112, 2735-52.

doi:10.1161/CIRCULATIONAHA.105.169404

Hirsch, J.A., Bishop, B., 1981. Respiratory sinus arrhythmia in humans: how breathing pattern modulates heart rate. Am. J. Physiol. Circ. Physiol. 241, H620-H629.

Hjemdahl, P., 2002. Stress and the metabolic syndrome an interesting but enigmatic association. Circulation 106, 2634-2636.

Hu, M.X., Lamers, F., de Geus, E.J.C., Penninx, B.W.J.H., n.d. Differential autonomic nervous system reactivity in depression and anxiety during stress depending on type of stressor. 
Mandy Xian Hu

Psychosom. Med. In Press.

Jorgensen, R.S., Nash, J.K., Lasser, N.L., Hymowitz, N., Langer, A.W., 1988. Heart Rate

Acceleration and its Relationship to Total Serum Cholesterol, Triglycerides, and Blood Pressure

Reactivity in Men with Mild Hypertension. Psychophysiology 25, 39-44. doi:10.1111/j.1469-

8986.1988.tb00955.x

Kimura, T., Matsumoto, T., Akiyoshi, M., Owa, Y., Miyasaka, N., Aso, T., Moritani, T., 2006. Body

fat and blood lipids in postmenopausal women are related to resting autonomic nervous system activity. Eur. J. Appl. Physiol. 97, 542-547.

Koskinen, T., Kähönen, M., Jula, A., Mattsson, N., Laitinen, T., Keltikangas-Järvinen, L., Viikari, J., Välimäki, I., Rönnemaa, T., Raitakari, O.T., 2009. Metabolic syndrome and short-term heart rate variability in young adults. Diabet. Med. 26, 354-361.

Kyrou, I., Tsigos, C., 2009. Stress hormones: physiological stress and regulation of metabolism. Curr. Opin. Pharmacol. 9, 787-793.

Lambert, E.A., Lambert, G.W., 2011. Stress and its role in sympathetic nervous system activation in hypertension and the metabolic syndrome. Curr. Hypertens. Rep. 13, 244-248.

Lambert, G.W., Straznicky, N.E., Lambert, E.A., Dixon, J.B., Schlaich, M.P., 2010. Sympathetic nervous activation in obesity and the metabolic syndrome - causes, consequences and therapeutic implications. Pharmacol. Ther. 126, 159-172.

Liao, D., Sloan, R.P., Cascio, W.E., Folsom, A.R., Liese, A.D., Evans, G.W., Cai, J., Sharrett, A.R., 1998. Multiple metabolic syndrome is associated with lower heart rate variability: the Atherosclerosis Risk in Communities Study. Diabetes Care 21, 2116-2122.

Licht, C., Geus, E. De, 2009. Depression is associated with decreased blood pressure, but antidepressant use increases the risk for hypertension. Hypertension 53, 631-638.

Licht, C.M.M., de Geus, E.J.C., Penninx, B.W.J.H., 2013. Dysregulation of the autonomic nervous system predicts the development of the metabolic syndrome. J. Clin. Endocrinol. Metab. 98, 2484-2493.

Licht, C.M.M., de Geus, E.J.C., Zitman, F.G., Hoogendijk, W.J.G., van Dyck, R., Penninx, B.W.J.H., 2008. Association between major depressive disorder and heart rate variability in the 
Netherlands Study of Depression and Anxiety (NESDA). Arch. Gen. Psychiatry 65, 1358-1367.

Licht, C.M.M., Penninx, B.W.J.H., de Geus, E.J.C., 2012. Effects of antidepressants, but not psychopathology, on cardiac sympathetic control: a longitudinal study.

Neuropsychopharmacology 37, 2487-2495.

Licht, C.M.M., Vreeburg, S.A., van Reedt Dortland, A.K.B., Giltay, E.J., Hoogendijk, W.J.G., DeRijk, R.H., Vogelzangs, N., Zitman, F.G., de Geus, E.J.C., Penninx, B.W.J.H., 2010. Increased sympathetic and decreased parasympathetic activity rather than changes in hypothalamic-pituitary-adrenal axis activity is associated with metabolic abnormalities. J. Clin. Endocrinol. Metab. 95, 2458-2466.

Lien, R., Neijts, M., Willemsen, G., de Geus, E.J.C., 2015. Ambulatory measurement of the ECG Twave amplitude. Psychophysiology 52, 225-237.

Light, K.C., Dolan, C.A., Davis, M.R., Sherwood, A., 1992. Cardiovascular responses to an active coping challenge as predictors of blood pressure patterns 10 to 15 years later. Psychosom. Med. 54, 217-230.

Ljung, T., Holm, G., Friberg, P., Andersson, B., Bengtsson, B., Svensson, J., Dallman, M., McEwen, B., Björntorp, P., 2000. The activity of the hypothalamic-pituitary-adrenal axis and the sympathetic nervous system in relation to waist/hip circumference ratio in men. Obes. Res. 8, 487-495.

Mancia, G., Parati, G., 2004. Office compared with ambulatory blood pressure in assessing response to antihypertensive treatment: a meta-analysis. J. Hypertens.

Masuo, K., Kawaguchi, H., Mikami, H., Ogihara, T., Tuck, M.L., 2003. Serum uric acid and plasma norepinephrine concentrations predict subsequent weight gain and blood pressure elevation. Hypertension 42, 474-80. doi:10.1161/01.HYP.0000091371.53502.D3

Matthews, K., Salomon, K., 2003. Cardiovascular reactivity to stress predicts future blood pressure in adolescence. Psychosom. Med. 65, 410-415.

Matthews, K.A., Katholi, C.R., McCreath, H., Whooley, M.A., Williams, D.R., Zhu, S., Markovitz, J.H., 2004. Blood pressure reactivity to psychological stress predicts hypertension in the CARDIA study. Circulation 110, 74-8. doi:10.1161/01.CIR.0000133415.37578.E4 
Mehler, B., Reimer, B., Coughlin, J.F., 2010. Physiological Reactivity to Graded Levels of Cognitive Workload across Three Age Groups: An On-Road Evaluation. Proc. Hum. Factors Ergon. Soc. Annu. Meet. 54, 2062-2066. doi:10.1177/154193121005402409

Menkes, M., Matthews, K., Krantz, D., 1989. Cardiovascular reactivity to the cold pressor test as a predictor of hypertension. Hypertension 14, 524-530.

Min, K.-B., Min, J.-Y., Paek, D., Cho, S.-I., 2008. The Impact of the Components of Metabolic Syndrome on Heart Rate Variability: Using the NCEP-ATP III and IDF Definitions. Pacing Clin. Electrophysiol. 31, 584-591.

Ming, E., Adler, G., Kessler, R., 2004. Cardiovascular reactivity to work stress predicts subsequent onset of hypertension: the Air Traffic Controller Health Change Study. Psychosom. Med. 66, 459-465.

Mortara, A., Sleight, P., Pinna, G.D., Maestri, R., Prpa, A., La Rovere, M.T., Cobelli, F., Tavazzi, L., 1997. Abnormal awake respiratory patterns are common in chronic heart failure and may prevent evaluation of autonomic tone by measures of heart rate variability. Circulation 96, 246252.

Muldoon, M.F., 1995. Effects of Acute Psychological Stress on Serum Lipid Levels, Hemoconcentration, and Blood Viscosity. Arch. Intern. Med. 155, 615. doi:10.1001/archinte.1995.00430060077009

Neijts, M., Van Lien, R., Kupper, N., Boomsma, D., Willemsen, G., Geus, E.J.C., 2014. Heritability of cardiac vagal control in 24-h heart rate variability recordings: Influence of ceiling effects at low heart rates. Psychophysiology 51, 1023-1036.

Nyklíček, I., Bosch, J.A., Amerongen, A.V.N., 2005. A generalized physiological hyperreactivity to acute stressors in hypertensives. Biol. Psychol. 70, 44-51.

Organization, W.H., 2009. Global Health Risks: Mortality and Burden of Disease Attributable to Selected Major Risks.

Penninx, B.W.J.H., Beekman, A.T.F., Smit, J.H., Zitman, F.G., Nolen, W.A., Spinhoven, P., Cuijpers, P., De Jong, P.J., Van Marwijk, H.W.J., Assendelft, W.J.J., 2008. The Netherlands Study of Depression and Anxiety (NESDA): rationale, objectives and methods. Int. J. Methods Psychiatr. 
Res. 17, 121-140.

Romijn, J., Fliers, E., 2005. Sympathetic and parasympathetic innervation of adipose tissue: metabolic implications. Curr. Opin. Clin. Nutr. Metab. Care 8, 440-444.

Salomon, K., Bylsma, L.M., White, K.E., Panaite, V., Rottenberg, J., 2013. Is blunted cardiovascular reactivity in depression mood-state dependent? A comparison of major depressive disorder remitted depression and healthy controls. Int. J. Psychophysiol. 90, 50-57.

Scalco, A.Z., Rondon, M.U., Trombetta, I.C., Laterza, M.C., Azul, J.B., Pullenayegum, E.M., Scalco, M.Z., Kuniyoshi, F., Wajngarten, M., Negrão, C., Lotufo-Neto, F., 2009. Muscle sympathetic nervous activity in depressed patients before and after treatment with sertraline. J. Hypertens. 27, 2429-2436.

Schmitz, J., Krämer, M., Tuschen-Caffier, B., Heinrichs, N., Blechert, J., 2011. Restricted autonomic flexibility in children with social phobia. J. child Psychol. psychiatry 52, 1203-1211.

SHEP Cooperative Research Group, 1991. Prevention of stroke by antihypertensive drug treatment in older persons with isolated systolic hypertension. Final results of the Systolic Hypertension in the Elderly Program (SHEP). Jama 265, 3255-3264.

Stein, P.K., Barzilay, J.I., Domitrovich, P.P., Chaves, P.M., Gottdiener, J.S., Heckbert, S.R., Kronmal, R. a, 2007. The relationship of heart rate and heart rate variability to non-diabetic fasting glucose levels and the metabolic syndrome: the Cardiovascular Health Study. Diabet. Med. 24, 855-63. doi:10.1111/j.1464-5491.2007.02163.x

Steptoe, A., Cropley, M., 2000. Persistent high job demands and reactivity to mental stress predict future ambulatory blood pressure. J. Hypertens. 18, 581-586.

Steptoe, A., Fieldman, G., Evans, O., Perry, L., 1996. Cardiovascular Risk and Responsivity to Mental Stress: The Influence of Age, Gender and Risk Factors. Eur. J. Cardiovasc. Prev. Rehabil. 3, 83-93. doi:10.1177/174182679600300112

Tannen, R., Weiner, M., Marcus, S., 2006. Simulation of the Syst-Eur randomized control trial using a primary care electronic medical record was feasible. J. Clin. Epidemiol. 59, 254-264.

Thorp, A.A., Schlaich, M.P., 2015. Relevance of Sympathetic Nervous System Activation in Obesity and Metabolic Syndrome. J. Diabetes Res. 
Tuomisto, M.T., 1997. Intra-arterial blood pressure and heart rate reactivity to behavioral stress in normotensive, borderline, and mild hypertensive men. Heal. Psychol. 16, 554-565.

Ulrich-Lai, Y.M., Herman, J.P., 2009. Neural regulation of endocrine and autonomic stress responses. Nat. Rev. Neurosci. 10, 397-409.

Vogelzangs, N., Suthers, K., 2007. Hypercortisolemic depression is associated with the metabolic syndrome in late-life. Psychoneuroendocrinology 32, 151-159.

Vrijkotte, T.G.M., van den Born, B.-J.H., Hoekstra, C.M.C.A., Gademan, M.G.J., van Eijsden, M., de Rooij, S.R., Twickler, M.T.B., 2015. Cardiac Autonomic Nervous System Activation and Metabolic Profile in Young Children: The ABCD Study. PLoS One 10, e0138302. doi:10.1371/journal.pone.0138302

Yi, C., Fleur, S. La, Fliers, E., Kalsbeek, A., 2010. The role of the autonomic nervous liver innervation in the control of energy metabolism. Biochim. Biophys. Acta - Mol. Basis Dis. 1802, 416-431. 
Table 1. Sample Characteristics at Baseline (n=1922)

Demographics

Age

Female sex (\%)

Education, years

Health factors

Smoking (\%)

Alcohol use

Non-drinker (\%)

Mild/moderate drinker (\%)

Heavy drinker (\%)

Physical activity, median 1000 METmin/week (IQR)

Cardiovascular disease (\%)

Use of heart medication (\%)

Antidepressant use (\%)

Resting respiration rate, breaths/min

Autonomic variables

Basal level values

$\mathrm{HR}$, beats/min

RSA, ms

PEP, ms

Cognitive challenge stress reactivity values

HR reactivity, $\Delta$ beats/min

$\mathrm{RSA}$ reactivity, $\Delta \mathrm{ms}$

$\mathrm{PEP}$ reactivity, $\Delta \mathrm{ms}$

Personal-emotional stress reactivity values

HR reactivity, $\Delta$ beats $/$ min

$\mathrm{RSA}$ reactivity, $\Delta \mathrm{ms}$

$\mathrm{PEP}$ reactivity, $\Delta \mathrm{ms}$

Metabolic syndrome components
$43.7 \pm 13.2$

66.5

$12.7 \pm 3.3$

30.7

31.4

57.7

10.9

$3.1(1.6-5.5)$

4.0

13.9

22.9

$16.5 \pm 1.3$

$70.0 \pm 9.6$

$46.1 \pm 24.7$

$122.1 \pm 17.8$

$3.49 \pm 3.66$

$-7.69 \pm 16.18$

$-2.52 \pm 5.29$

$3.60 \pm 3.37$

$-4.51 \pm 7.95$

$-2.41 \pm 6.06$ 
Number of metabolic components, median (IQR)

0 metabolic components (\%)

1-2 metabolic components (\%)

$\geq 3$ metabolic components (\%)

Triglycerides, mmol/L

HDL cholesterol, mmol/L

Systolic blood pressure, mm Hg

Diastolic blood pressure, mm Hg

Glucose, $\mathrm{mmol} / \mathrm{L}$

Waist circumference, $\mathrm{cm}$
$1.00(0.00-2.00)$

27.3

49.7

23.1

$1.30 \pm 0.95$

$1.56 \pm 0.42$

$132.89 \pm 18.35$

$79.19 \pm 10.78$

$5.31 \pm 0.97$

$89.00 \pm 13.93$

Note: Values represent mean \pm SD unless otherwise indicated. Metmin $=$ multiple of resting metabolic rate times minutes of physical activity per week. IQR = interquartile range. $\mathrm{HR}=$ heart rate. RSA = respiratory sinus arrhythmia. $\mathrm{PEP}=$ pre-ejection period. $\mathrm{HDL}=$ high density lipoprotein.

Basal ANS values were obtained by taking the average score of n-back rest and the general interview. Stress reactivity values represent ANS values during cognitive challenge (n-back task) or personal-emotional stress (psychiatric interview) minus values during n-back rest or the general interview respectively. 
Mandy Xian $\mathrm{Hu}$

Table 2. Adjusted Associations between Autonomic Basal and Stress Reactivity Values and Individual Components of the Metabolic Syndrome

\begin{tabular}{|c|c|c|c|c|c|c|c|c|c|c|c|c|c|c|c|c|c|c|c|}
\hline & & \multirow{2}{*}{\multicolumn{3}{|c|}{$\begin{array}{l}\text { No. of MetS components } \\
\qquad \mathrm{N}=1796\end{array}$}} & \multirow{2}{*}{\multicolumn{3}{|c|}{$\begin{array}{l}\text { Triglycerides, mmol/L } \\
\qquad \mathrm{N}=1839\end{array}$}} & \multirow{2}{*}{\multicolumn{3}{|c|}{$\begin{array}{c}\text { HDL cholesterol, mmol/L } \\
\qquad \mathrm{N}=1843\end{array}$}} & \multirow{2}{*}{\multicolumn{3}{|c|}{$\begin{array}{c}\text { SBP, mm Hg } \\
\quad \mathrm{N}=1917\end{array}$}} & \multirow{2}{*}{\multicolumn{3}{|c|}{$\begin{array}{c}\text { Glucose, } \mathbf{~ m m o l} / \mathbf{L} \\
\qquad \mathrm{N}=1855\end{array}$}} & \multirow{2}{*}{\multicolumn{3}{|c|}{$\begin{array}{l}\text { Waist circumference, } \mathbf{c m} \\
\qquad \mathrm{N}=1897\end{array}$}} \\
\hline & & & & & & & & & & & & & & & & & & & \\
\hline & & $\boldsymbol{\beta}$ & $\mathbf{p}$ & $\mathbf{R}^{2}$ & $\boldsymbol{\beta}$ & $\mathbf{p}$ & $\overline{\mathbf{R}^{2}}$ & $\boldsymbol{\beta}$ & $\mathbf{p}$ & $\mathbf{R}^{2}$ & $\boldsymbol{\beta}$ & $\mathbf{p}$ & $\overline{\mathbf{R}^{2}}$ & $\beta$ & $\mathbf{p}$ & $\mathbf{R}^{2}$ & $\beta$ & $\mathbf{p}$ & $\overline{\mathbf{R}^{2}}$ \\
\hline Model 1 & HR basal, bpm & .128 & $<.001$ & .232 & .152 & $<.001$ & .148 & -.038 & .081 & .170 & .118 & $<.001$ & .275 & .108 & $<.001$ & .211 & .106 & $<.001$ & .268 \\
\hline \multirow[t]{3}{*}{ Model 2} & HR basal, bpm & .125 & $<.001$ & & .149 & $<.001$ & & -.032 & .14 & & .117 & $<.001$ & & .106 & $<.001$ & & .105 & $<.001$ & \\
\hline & $\mathrm{HR}$ cognitive challenge reactivity, $\Delta \mathrm{bpm}$ & -.033 & 12 & .233 & -.026 & .25 & .149 & .056 & .012 & .174 & .024 & .25 & .276 & -.034 & .11 & .212 & -.055 & .008 & .271 \\
\hline & HR personal-emotional reactivity, $\Delta \mathrm{bpm}$ & -.016 & .45 & & -.011 & .61 & & .031 & .16 & & -.021 & .28 & & .003 & .87 & & .017 & .41 & \\
\hline Model 1 & RSA basal, ms & -.071 & .005 & .229 & -.059 & .027 & .134 & .031 & .23 & .176 & -.079 & .001 & .268 & -.046 & .071 & .205 & -.054 & .024 & .270 \\
\hline \multirow[t]{3}{*}{ Model 2} & RSA basal, ms & -.141 & $<.001$ & & -.115 & $<.001$ & & .047 & .13 & & -.126 & $<.001$ & & -.088 & .004 & & -.125 & $<.001$ & \\
\hline & RSA cognitive challenge reactivity, $\Delta \mathrm{ms}$ & -.102 & $<.001$ & .237 & -.091 & $<.001$ & .140 & .048 & .055 & .179 & -.071 & .002 & .272 & -.038 & .12 & .208 & -.089 & $<.001$ & .277 \\
\hline & RSA personal-emotional reactivity, $\Delta \mathrm{ms}$ & -.032 & .16 & & -.012 & .62 & & -.025 & .27 & & -.014 & .50 & & -.051 & .023 & & -.051 & .017 & \\
\hline Model 1 & PEP basal, ms & -.127 & $<.001$ & .232 & -.090 & $<.001$ & .134 & .055 & .009 & .172 & -.120 & $<.001$ & .276 & -.015 & .49 & .200 & -.141 & $<.001$ & .277 \\
\hline \multirow[t]{3}{*}{ Model 2} & PEP basal, ms & -.134 & $<.001$ & & -.094 & $<.001$ & & .065 & .003 & & -.126 & $<<.001$ & & -.019 & .39 & & -.147 & $<.001$ & \\
\hline & PEP cognitive challenge reactivity, $\Delta \mathrm{ms}$ & -.030 & .15 & .234 & -.038 & .085 & .135 & -.010 & .64 & .174 & -.040 & .039 & .278 & -.027 & .21 & .201 & -.013 & .51 & .278 \\
\hline & PEP personal-emotional reactivity, $\Delta \mathrm{ms}$ & -.019 & .37 & & -.003 & .90 & & .044 & .046 & & -.010 & .61 & & -.009 & .69 & & -.018 & .38 & \\
\hline
\end{tabular}


Mandy Xian Hu

Table 3. Longitudinal Changes in Metabolic Syndrome Components ( $\mathrm{n}=1616)$

\begin{tabular}{llcc}
\hline & Baseline & 4-year follow-up & $\Delta$ \\
\hline Number of metabolic components, median (IQR) & $1.0(0.00-2.00)$ & $1.00(0.00-3.00)$ & $0.17 \pm 1.00$ \\
Triglycerides, mmol/L & $1.28 \pm 0.90$ & $1.29 \pm 0.86$ & $0.01 \pm 0.72$ \\
HDL cholesterol, mmol/L & $1.55 \pm 0.42$ & $1.56 \pm 0.43$ & $0.01 \pm 0.26$ \\
Systolic blood pressure, mm Hg & $132.70 \pm 18.04$ & $134.30 \pm 19.55$ & $1.60 \pm 12.69$ \\
Diastolic blood pressure, mm Hg & $78.98 \pm 10.65$ & $79.36 \pm 10.74$ & $0.38 \pm 7.66$ \\
Glucose, mmol/L & $5.30 \pm 0.90$ & $5.52 \pm 1.04$ & $0.22 \pm 0.72$ \\
Waist circumference, cm & $89.16 \pm 13.92$ & $92.00 \pm 14.07$ & $2.82 \pm 7.63$ \\
\hline Note: Values represent mean \pm SD unless otherwise indicated. IQR = interquartile range. HDL = high \\
density lipoprotein.
\end{tabular}


Mandy Xian Hu

Table 4. Adjusted Associations between Autonomic Basal and Stress Reactivity Values and 4-year Changes in Individual Components of the Metabolic Syndrome

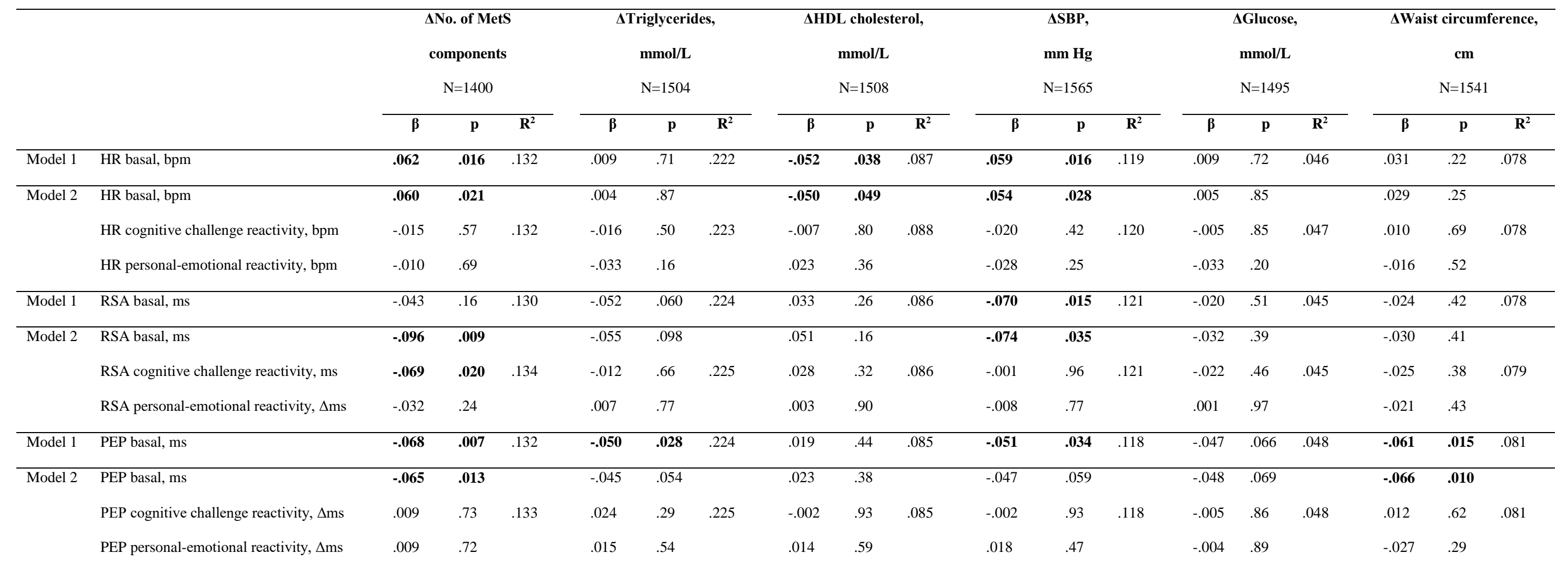

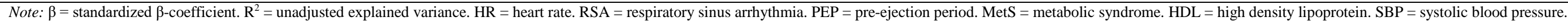
Triglycerides and glucose values were ln-transformed for analyses. Significant results at $\mathrm{p}<.05$ and significant changes in $\mathrm{R}^{2}$ are highlighted with bold formatting.

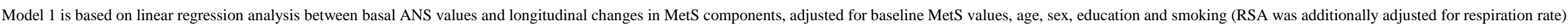
In model 2 ANS stress reactivity values during a cognitively challenging stressor and during a personal-emotional stressor were added to model 1 as predictors. 
Mandy Xian Hu

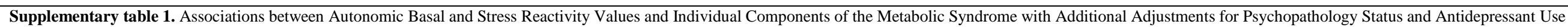

\begin{tabular}{|c|c|c|c|c|c|c|c|c|c|c|c|c|c|c|c|c|c|c|}
\hline & \multirow{2}{*}{\multicolumn{3}{|c|}{$\begin{array}{l}\text { No. of MetS components } \\
\qquad \mathrm{N}=1796\end{array}$}} & \multirow{2}{*}{\multicolumn{3}{|c|}{$\begin{array}{c}\text { Triglycerides, } \mathbf{m m o l} / \mathbf{L} \\
\text { N=1839 }\end{array}$}} & \multirow{2}{*}{\multicolumn{3}{|c|}{$\begin{array}{l}\text { HDL cholesterol, } \\
\qquad \begin{array}{l}\text { mmol/L } \\
\mathrm{N}=1843\end{array}\end{array}$}} & \multirow{2}{*}{\multicolumn{3}{|c|}{$\begin{array}{l}\text { SBP, } \mathbf{m m ~ H g} \\
\quad \mathrm{N}=1917\end{array}$}} & \multirow{2}{*}{\multicolumn{3}{|c|}{$\begin{array}{c}\text { Glucose, } \mathbf{~ m m o l} / \mathbf{L} \\
\qquad \mathrm{N}=1855\end{array}$}} & \multirow{2}{*}{\multicolumn{3}{|c|}{$\begin{array}{l}\text { Waist circumference, } \mathbf{c m} \\
\qquad \mathrm{N}=1897\end{array}$}} \\
\hline & & & & & & & & & & & & & & & & & & \\
\hline & $\boldsymbol{\beta}$ & $\mathbf{p}$ & $\overline{\mathbf{R}^{2}}$ & $\beta$ & $\mathbf{p}$ & $\overline{\mathbf{R}^{2}}$ & $\boldsymbol{\beta}$ & $\mathbf{p}$ & $\overline{\mathbf{R}^{2}}$ & $\bar{\beta}$ & $\mathbf{p}$ & $\mathbf{R}^{2}$ & $\boldsymbol{\beta}$ & $\mathbf{p}$ & $\overline{\mathbf{R}^{2}}$ & $\boldsymbol{\beta}$ & $\mathbf{p}$ & $\overline{\mathbf{R}^{2}}$ \\
\hline HR basal, bpm & .121 & $<.001$ & & .152 & $<.001$ & & -.024 & .28 & & .106 & $<.001$ & & .109 & $<.001$ & & .096 & $<.001$ & \\
\hline $\mathrm{HR}$ cognitive challenge reactivity, $\Delta \mathrm{bpm}$ & -.030 & .17 & .239 & -.020 & .37 & .155 & .053 & .016 & .178 & .019 & .35 & .279 & -.030 & .16 & .214 & -.049 & .016 & .284 \\
\hline HR personal-emotional reactivity, $\Delta \mathrm{bpm}$ & -.021 & .33 & & -.016 & .47 & & .035 & .11 & & -.014 & .47 & & .003 & .88 & & .008 & .70 & \\
\hline RSA basal, ms & -.117 & $<.001$ & & -.092 & .005 & & .028 & .38 & & -.118 & $<.001$ & & -.081 & .010 & & -.086 & .003 & \\
\hline RSA cognitive challenge reactivity, $\Delta \mathrm{ms}$ & -.097 & $<.001$ & .241 & -.088 & .001 & .145 & .044 & .076 & .182 & -.065 & .005 & .277 & -.038 & .12 & .210 & -.082 & $<.001$ & .290 \\
\hline RSA personal-emotional reactivity, $\Delta \mathrm{ms}$ & -.027 & .24 & & -.007 & .77 & & -.030 & .20 & & -.019 & .38 & & -.048 & .034 & & -.042 & .049 & \\
\hline PEP basal, ms & -.133 & $<.001$ & & -.096 & $<.001$ & & .058 & .012 & & -.123 & $<.001$ & & -.014 & .55 & & -.141 & $<.001$ & \\
\hline PEP cognitive challenge reactivity, $\Delta \mathrm{ms}$ & -.031 & .14 & .239 & -.038 & .082 & .142 & -.010 & .63 & .177 & -.039 & .048 & .282 & -.027 & .20 & .203 & -.013 & .52 & .290 \\
\hline PEP personal-emotional reactivity, $\Delta \mathrm{ms}$ & -.020 & .36 & & -.003 & .88 & & .043 & .048 & & -.012 & .56 & & -.008 & .71 & & -.017 & .40 & \\
\hline
\end{tabular}

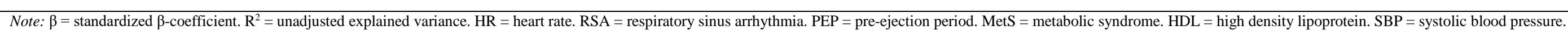

Triglycerides and glucose values were ln-transformed for analyses. Significant results at $\mathrm{p}<.05$ are highlighted with bold formatting.

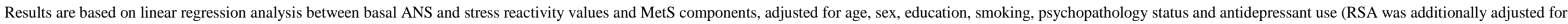
respiration rate). 
Mandy Xian Hu

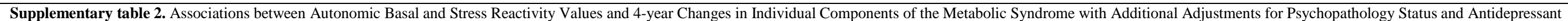
Use

\begin{tabular}{|c|c|c|c|c|c|c|c|c|c|c|c|c|c|c|c|c|c|c|}
\hline & \multicolumn{3}{|c|}{$\begin{array}{c}\text { No. of MetS components } \\
\qquad N=1400\end{array}$} & \multirow{2}{*}{\multicolumn{3}{|c|}{$\begin{array}{c}\Delta \text { Triglycerides, } \\
\text { mmol/L } \\
\mathrm{N}=1504\end{array}$}} & \multirow{2}{*}{\multicolumn{3}{|c|}{$\begin{array}{c}\text { SHDL cholesterol, } \\
\text { mmol/L } \\
\mathrm{N}=1508\end{array}$}} & \multirow{2}{*}{\multicolumn{3}{|c|}{$\begin{array}{l}\Delta \text { SBP, } \\
\text { mm Hg } \\
\mathrm{N}=1565\end{array}$}} & \multirow{2}{*}{\multicolumn{3}{|c|}{$\begin{array}{c}\begin{array}{c}\Delta \text { Glucose, } \\
\text { mmol/L } \\
\mathrm{N}=1495\end{array}\end{array}$}} & \multirow{2}{*}{\multicolumn{3}{|c|}{$\begin{array}{c}\Delta \text { Waist circumference, } \\
\text { cm } \\
\mathrm{N}=1541\end{array}$}} \\
\hline & \multirow[b]{2}{*}{$\beta$} & \multirow[b]{2}{*}{$\mathbf{p}$} & \multirow[b]{2}{*}{$\mathbf{R}^{2}$} & & & & & & & & & & & & & & & \\
\hline & & & & $\boldsymbol{\beta}$ & $\mathbf{p}$ & $\mathbf{R}^{2}$ & $\beta$ & $\mathbf{p}$ & $\mathbf{R}^{2}$ & $\boldsymbol{\beta}$ & $\mathbf{p}$ & $\mathbf{R}^{2}$ & $\boldsymbol{\beta}$ & $\mathbf{p}$ & $\mathbf{R}^{2}$ & $\boldsymbol{\beta}$ & $\mathbf{p}$ & $\mathbf{R}^{2}$ \\
\hline HR basal, bpm & .066 & .014 & & -.004 & .86 & & -.054 & .038 & & .053 & .036 & & -.001 & .97 & & .032 & .22 & \\
\hline HR cognitive challenge reactivity, bpm & -.011 & .67 & .135 & -.015 & .54 & .226 & -.007 & .78 & .089 & -.021 & .40 & .121 & -.005 & .87 & .051 & .008 & .75 & .083 \\
\hline HR personal-emotional reactivity, bpm & -.013 & .62 & & -.037 & .12 & & .022 & .39 & & -.027 & .28 & & -.036 & .17 & & -.014 & .58 & \\
\hline RSA basal, ms & -.095 & .013 & & $\begin{array}{l}-.043 \\
\end{array}$ & .22 & & .054 & .15 & & -.078 & .033 & & $\begin{array}{l}.024 \\
\end{array}$ & .53 & & -.052 & .16 & \\
\hline RSA cognitive challenge reactivity, ms & -.070 & .017 & .137 & -.009 & .74 & .227 & .029 & .32 & .087 & -.002 & .95 & .123 & -.021 & .48 & .049 & -.029 & .31 & .085 \\
\hline RSA personal-emotional reactivity, $\Delta \mathrm{ms}$ & -.031 & .26 & & -.010 & .68 & & .005 & .87 & & -.010 & .71 & & -.002 & .94 & & -.016 & .55 & \\
\hline PEP basal, ms & $\begin{array}{l}.074 \\
\end{array}$ & .007 & & -.037 & .14 & & .028 & .30 & & -.049 & .059 & & -.045 & .11 & & $\begin{array}{c}.071 \\
\end{array}$ & .009 & \\
\hline PEP cognitive challenge reactivity, $\Delta \mathrm{ms}$ & .010 & .70 & .136 & .024 & .30 & .227 & -.003 & .92 & .086 & -.002 & .93 & .119 & -.004 & .87 & .052 & .013 & .61 & .086 \\
\hline PEP personal-emotional reactivity, $\Delta \mathrm{ms}$ & .007 & .78 & & .016 & .50 & & .015 & .55 & & .016 & .51 & & -.004 & .89 & & -.027 & .29 & \\
\hline
\end{tabular}

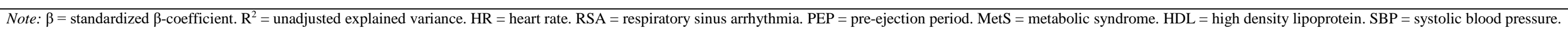

Triglycerides and glucose values were ln-transformed for analyses. Significant results at $\mathrm{p}<.05$ are highlighted with bold formatting.

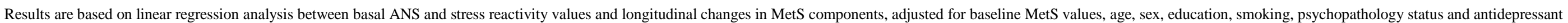
use (RSA was additionally adjusted for respiration rate) 


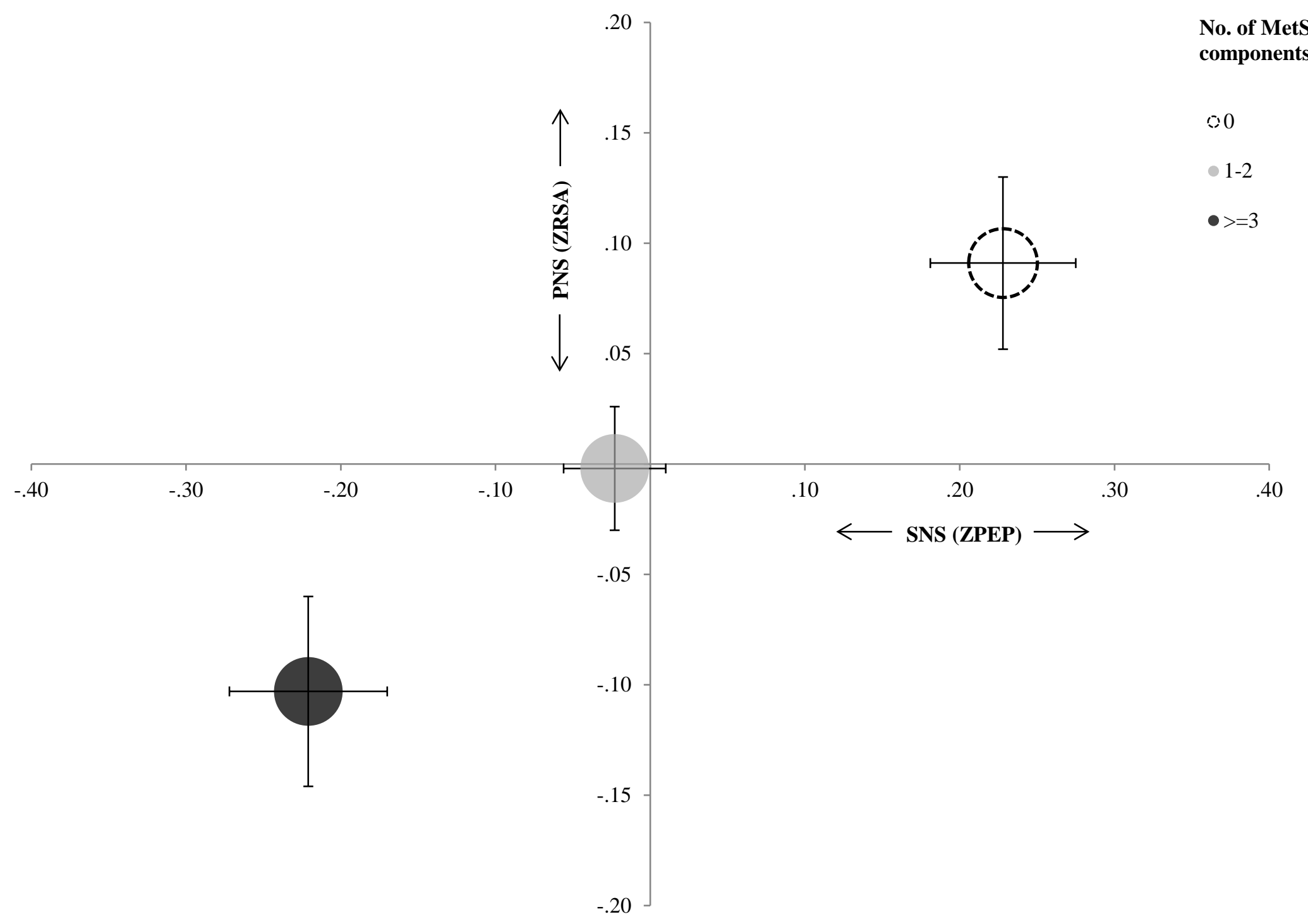

Figure 1. Mean z-score and standard error (represented by error bars) for RSA and PEP after adjusting for age, sex, education and smoking (RSA was additionally adjusted for respiration rate), depicted for participants with no MetS components ("0"), intermediate number of MetS components ("1-2") and high number of MetS components (">=3"). 